\title{
Role of iron oxide impurities in electrocatalysis by multiwall carbon nanotubes: An investigation using a novel magnetically modified ITO electrodes
}

\author{
KANCHAN M SAMANT, VRUSHALI S JOSHI, KASHINATH R PATIL ${ }^{\dagger}$ \\ and SANTOSH K HARAM* \\ Department of Chemistry, University of Pune, Ganeshkhind, Pune 411 007, India \\ $\dagger$ Physical and Material Chemistry Division, National Chemical Laboratory, Pune 411 008, India
}

MS received 9 July 2012; revised 28 December 2012

\begin{abstract}
The role of iron oxide impurities in the electrocatalytic properties of multiwall carbon nanotubes (MWCNTs) prepared by catalytic chemical vapour decomposition method (CCVD) is studied in detail. A novel magnetically modified electrodes have been developed by which MWCNTs were immobilized on indium-tin oxide (ITO) electrodes, without any chemical binders. The electro-catalytic oxidation of dopamine, and reduction of hydrogen peroxide have been studied by cyclic voltammetry on magnetically modified electrodes with (i) MWCNTs with occluded iron oxide impurities (Fe-MWCNTs), (ii) MWCNTs grown on iron oxide nanoparticle particulate films (Io-MWCNTs) and (iii) pristine iron oxide nanoparticle particulate film (Io-NPs). A shift towards less positive potentials for the oxidation of dopamine was observed which is in the order of Fe-MWCNTs < Io-MWCNTs < Io-NPs. Similarly, trend towards less negative potentials for the reduction of hydrogen peroxide was observed. Thus, the electrocatalytic activities displayed by MWCNTs have been attributed to the iron oxide impurities associated with it. The systematic variation was related to the nature of interaction of iron oxide nanoparticles with MWCNT surface.
\end{abstract}

Keywords. Multiwall carbon nanotubes (MWCNTs); iron oxide nanoparticles; dopamine; hydrogen peroxide; magnetically-modified electrode.

\section{Introduction}

Due to the good electrical conductivity, chemical inertness and well defined crystalline surface, carbon nanotubes (CNTs) have been viewed as a promising electrode material for the amperometric biosensor applications (Zhao et al 2002). Britto et al (1999) were among the first, to demonstrate the possibility of CNT modified electrode in the dopamine sensing applications. These electrodes decrease the overpotential for NADH oxidation reaction (Musameh et al 2002) and also can be used for the amperometric detection of hydrogen peroxide (Wang et al 2003), ascorbic acid, uric acid, dopamine and dopac (Rubianes and Rivas 2003). The electrochemical behaviour of single wall carbon nanotubes (SWCNTs) paste electrode has been reported and was further tested as $\mathrm{H}_{2} \mathrm{O}_{2}$ and $\mathrm{NADH}$ probes (Valentini et al 2003). All these reports in this regard have been converged to the conclusion that the electrocatalytic activity shown by CNTs is a complex response of distinct sites present on CNTs viz. basal plane, edge plane and metal/metal oxide impurities. There are very few reports, which suggested that, the edge planes are responsible for NADH biosensing properties (Gooding et al 2003; Banks and Compton 2005). In another work, it is reported that the metallic impurities in the form of

*Author for correspondence (haram@chem.unipune.ac.in) oxides, entrapped during catalytic chemical vapour decomposition (CCVD) growth are responsible for the electrocatalysis shown by CNTs (Sljukic et al 2006; Jones et al 2007). Nevertheless, very less attention has been paid so far to establish the crystallographic phase and oxidation state of the metal impurities present in MWCNTs and their contribution in the electrocatalysis.

Herein, we prepared MWCNTs by CCVD method with ferrocene as a catalyst, which led to the formation of MWCNTs occluded with iron oxide impurity. For the comparative studies, we prepared MWCNTs in which CVD process was catalyzed by the well-characterized iron oxide particulate films. This method of preparation provided MWCNTs grown on a substrate and gave rather physical mixture of MWCNTs and iron oxide which is distinctly different from the occluded case. All the samples have inherent ferromagnetic properties at room temperature, which have been used to prepare novel magnetically modified ITO electrodes. This novelty in the electrode preparation totally avoided the use of external binders such as nafion (Chicharro et al 2005), teflon (Wang and Musameh 2003), bromoform (Britto et al 1999), mineral oil (Rubianes and Rivas 2003), polymer matrix (Maogen and Waldemar 2005) to hold the sample firmly on ITO during the electrochemical measurements. The added advantage of this strategy is that possible electrochemical-interference from these binding materials 
could be completely avoided. Thus, a true representation of the properties imparted by MWCNTs/iron oxide surface, either individually or together could be highlighted. The electrochemical behaviour of dopamine and hydrogen peroxide has been chosen to investigate the performance of the modified electrodes. Using these set of experiments, we tried to bring more clarity in the electrocatalytic activities normally attributed to MWCNTs modified electrodes.

\section{Experimental}

\subsection{Materials}

Ferrocene (Fluka), naphthalene (s.d. fine), benzene (Merck), xylene (s.d. fine), potassium chloride (s.d. fine) and all the other chemicals were of analytical reagent grade and used asreceived without further purification. Ar gas with the highest possible purity $\left(\mathrm{O}_{2}<2 \mathrm{ppm}\right)$, was procured from India Oxygen Limited. All the solutions were prepared in Mill-Q water $\left(18 \mathrm{M} \Omega \mathrm{cm}^{-1}\right)$.

\subsection{Preparation of MWCNTs}

MWCNTs samples used in these experiments were prepared by catalytic chemical vapour decomposition (CCVD) method, in presence of two different starting catalyst materials, viz. ferrocene and $\mathrm{Fe}_{2} \mathrm{O}_{3}$ particulate films (hereafter Fe-MWCNTs and Io-MWCNTs, respectively). The experimental conditions and setup used for the preparation of FeMWCNTs are described in details by our group, previously (Samant et al 2007). On the basis of Raman spectroscopy, powder X-ray diffraction analysis (XRD), scanning electron microscopy (SEM), the formation of MWCNTs having an average diameter of ca. $64 \pm 5 \mathrm{~nm}$ was inferred (Samant et al 2007). For Io-MWCNTs, the nanotubes were grown on $\mathrm{Fe}_{2} \mathrm{O}_{3}$ particulate films which was predeposited on fused silica substrate, by the flame pyrolysis method (Inamdar and Haram 2006). In brief, spontaneous combustion of solution of ferrocene in ethanol gave iron oxide nanoparticles (Io-NPs) with an average diameter of about $24.7 \pm 1.6 \mathrm{~nm}$ deposited on either fused silica or ITO glass substrates. The fused silica substrate coated with Io-NPs was used for the synthesis of MWCNTs by CCVD method.

\subsection{Characterization techniques}

Powder X-ray diffractograms (XRD) on the vacuum dried products were recorded using Brucker D8 advanced diffractometer, $(\mathrm{CuK} \alpha, 40 \mathrm{kV}, 40 \mathrm{~mA})$. SEM images were recorded to understand the morphology of the product using JEOL JSM-6360 electron microscope. To investigate the surface oxidation state of iron oxide in the samples, XPS analysis was carried out using ESCA-3000 (VG Scientific Ltd, England) with a base pressure $10^{-9} \mathrm{~Pa}$. AlK $\alpha$ source $(1486 \cdot 6 \mathrm{eV})$, operated at $150 \mathrm{~W}$ was used as a X-ray source. The binding energy values were charge-corrected to $\mathrm{C} 1 s$ signal (284.6 eV). Vibrating sample magnetometer (VSM), model LakeShore 7307 was used for the magnetic measurements of the samples. All the measurements were carried out at room temperature.

\subsection{Fabrication of MWCNTs-modified magnet electrode $(M M E)$}

The magnetically modified electrode is schematically represented in figure 1. ITO-coated borosilicate glass was used as a conducting substrate for the preparation of MME. For that, ITO $(2 \times 1 \mathrm{~cm})$ was sonicated in triethanol amine, rinsed with copious amount of Milli-Q water and dried under vacuum. A small permanent magnet $(2 \times 5 \mathrm{~mm})$ was fixed firmly on the non-conducting side of ITO, using a silicone adhesive (Dow Corning, USA). Electrical contact to ITO substrate was made through copper wire coated with a silver paint. Entire electrode was masked with a room temperature vulcanizing (RTV) silicone adhesive, leaving a small window (ca. $0.25 \mathrm{~cm}^{2}$ ) on a conducting face just opposite to the magnet for the electrochemical contact. One milligram of the sample was sprinkled carefully on the exposed window of the electrode which would be spontaneously adhered to the conducting side of the electrode due to its magnetic properties. All the voltammetric investigations were done on these MMEs.

\subsection{Voltammetric measurements}

Voltammetric measurements were carried out with the help of potentiostat/galvanostat model autolab PGSTAT100

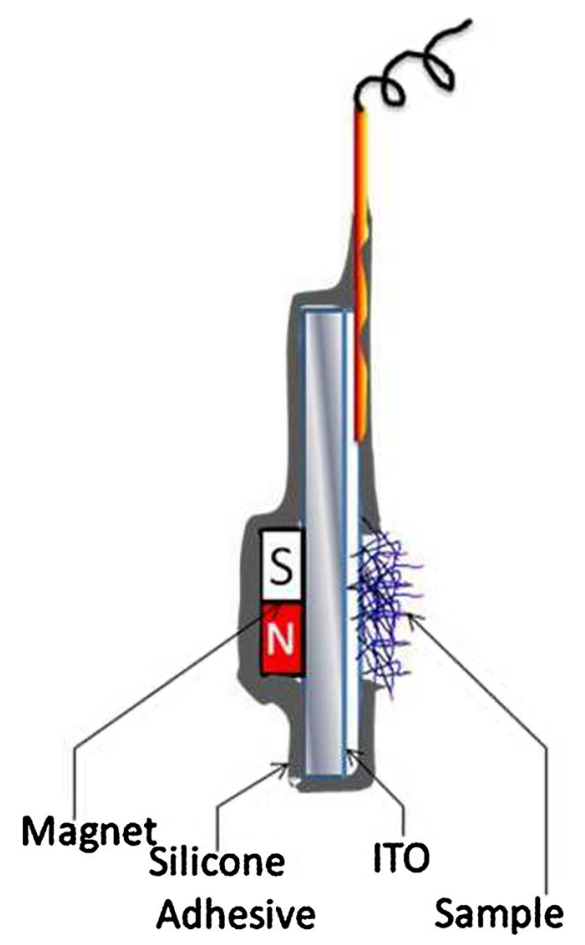

Figure 1. Schematics of magnetically modified electrode (MME). 
(Metrohm, Netherlands). The conventional three-electrode system was used, in $0.1 \mathrm{M} \mathrm{KCl}$ having $\mathrm{Pt}$ rod and $\mathrm{Ag} / \mathrm{AgCl}$ (3 $\mathrm{M} \mathrm{KCl}$ ), as counter and reference electrodes, respectively. All the measurements were carried out in an argon atmosphere and at room temperature.

\section{Results and discussion}

\subsection{Material characterization of MWCNTs}

Representative, SEM images recorded on the samples, Fe-MWCNTs, Io-MWCNTs and Io-NPs are shown in figures 2(a), (b) and (c), respectively. In case of $\mathrm{Fe}$ MWCNTs, entangled bundles of carbon nanotubes with an average outer diameter of $64 \pm 5 \mathrm{~nm}$ are legible. A close inspection of the image revealed the presence of iron oxide nanoparticles occluded at some places (marked as dotted circles). The micrograph of Io-MWCNTs however, showed straight thread-like nanotubes having diameter $25 \pm 5 \mathrm{~nm}$. In this case, the occluded nanoparticles were not apparent in the micrograph. This observation is obvious as MWCNTs were grown on iron-oxide modified films (Io-NPs). Figure 2(c) shows SEM image for Io-NPs prepared by flame pyrolysis method. It shows clusters of particles, which led to the particulate film. These samples were used directly without any further treatment in the fabrication of MMEs.

XRD recorded on Fe-MWCNTs, Io-MWCNTs and IoNPs are shown in figures 3(a), (b) and (c), respectively. The peaks at $26.5,42.9,54.7$ and $77.9^{\circ}$ (marked by “*”) in case of Fe-MWCNTs and Io-MWCNTs are attributed to the diffractions from (002), (100), (004) and (110) planes of the $s p^{2}$ hybridized hexagonal graphitic structures ( $\mathrm{Li}$ et al 2003). Other reflections observed in all the three samples at $30 \cdot 2,35 \cdot 6,43 \cdot 2,53 \cdot 7,57 \cdot 2$ and $62.9^{\circ}$ matched with the one reported for $\gamma-\mathrm{Fe}_{2} \mathrm{O}_{3}$ phase (JCPDS-39-1346). These observations suggested that even though the occluded iron oxide in MWCNTs is not legible in SEM of Io-MWCNTs (see figure 2c), these are still present in the samples. Thus, IoMWCNTs is a physical mixture of MWCNTs and iron oxide in which MWCNTs are covered over iron oxide film.

The valence state of iron and the surface composition of the sample were estimated by X-ray photo-electron spectroscopy (XPS). The survey scans recorded on these samples are given in the supporting information. Figures 4(a), (b) and (c), depict $2 p$ core level spectra in case of Fe-MWCNTs, Io-MWCNTs and Io-NPs samples, respectively. The binding energies of $2 p^{3 / 2}$ observed in all the cases are in the range of $710-711 \mathrm{eV}$, which correspond to $\mathrm{Fe}^{3+}$ valence state. A satellite structures observed in all the cases indicated the presence of $\mathrm{Fe}_{2} \mathrm{O}_{3}$ (Fujii et al 1999). A relatively weak peaks of $\mathrm{Fe}_{2} \mathrm{O}_{3}$ in case of Io-MWCNTs suggested less amount of iron oxide exposed on the surface of Io-MWCNTs irrespective of their bulk proportion (refer XRD peak height in figure 3 ). This observation is also in agreement with SEM analysis in which MWCNTs covered over iron oxide film was seen.
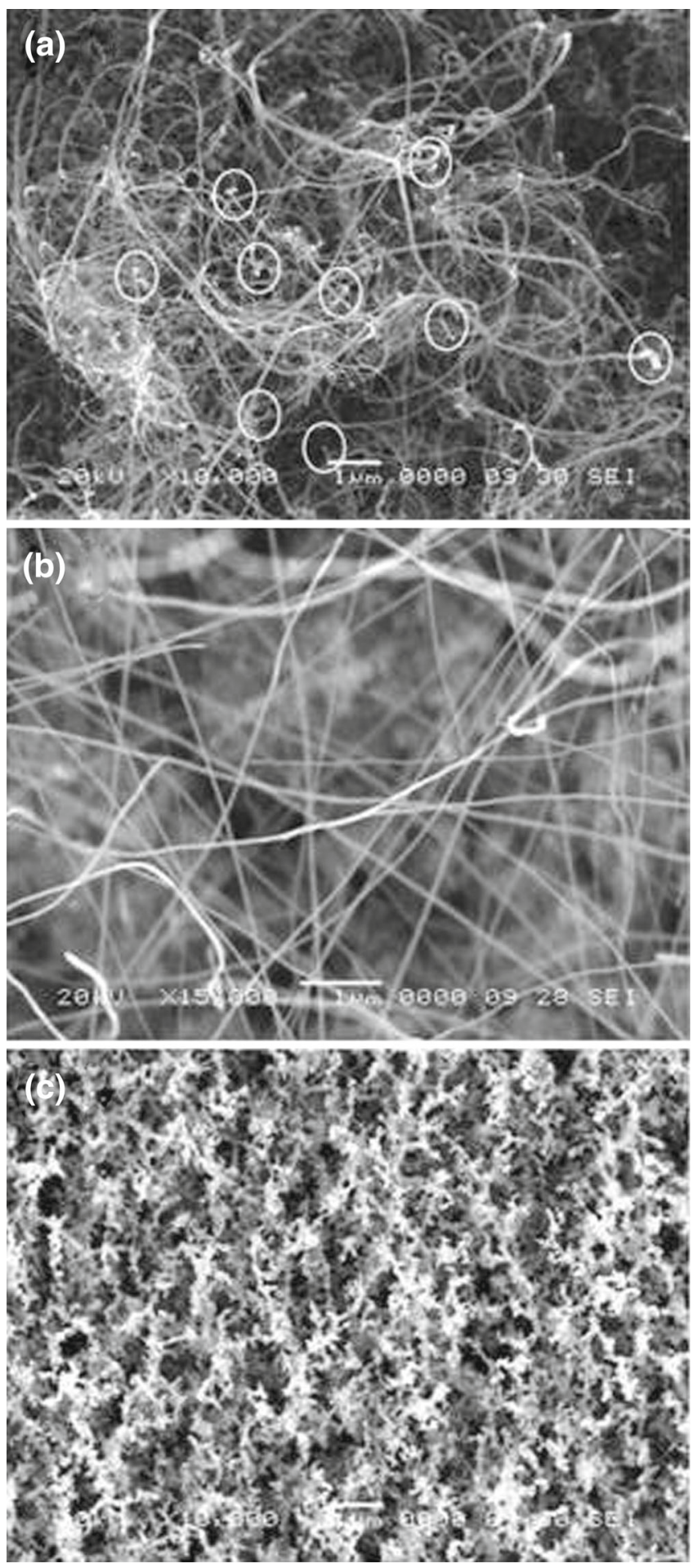

Figure 2. SEM images recorded on the samples. (a) MWCNTs prepared by CCVD method using ferrocene as catalyst (FeMWCNTs). The occluded iron oxide impurities legible in the micrograph (marked as dashed circle). (b) MWCNTs grown on the substrate with iron oxide particulate film (Io-MWCNTs). (c) Iron oxide film (Io-NPs) prepared by flame pyrolysis.

The iron oxide impurities remained incorporated during synthesis, imparted magnetism to MWCNTs. These were investigated at room temperature using vibrating sample 


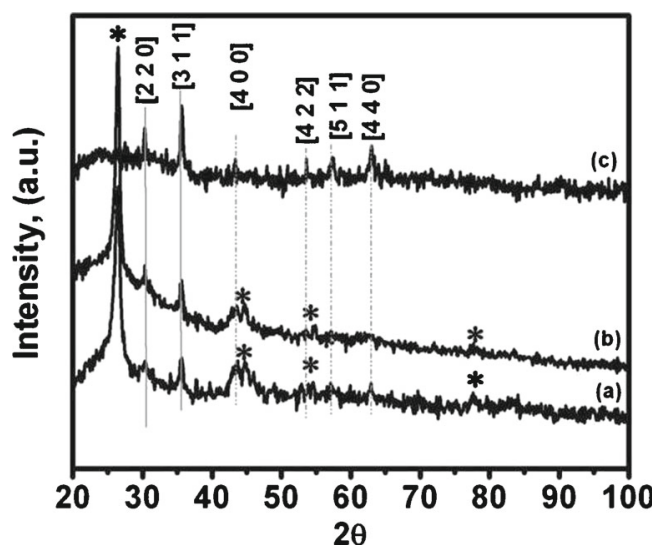

Figure 3. XRDs recorded on (a) Fe-MWCNTs, (b) Io-MWCNTs and (c) Io-NPs. The reflections marked with "*” are the graphitic reflections in MWCNTs. Rest of the peaks are fitted faithfully in $\gamma$-iron oxide phase (JCPDS 39-1346).
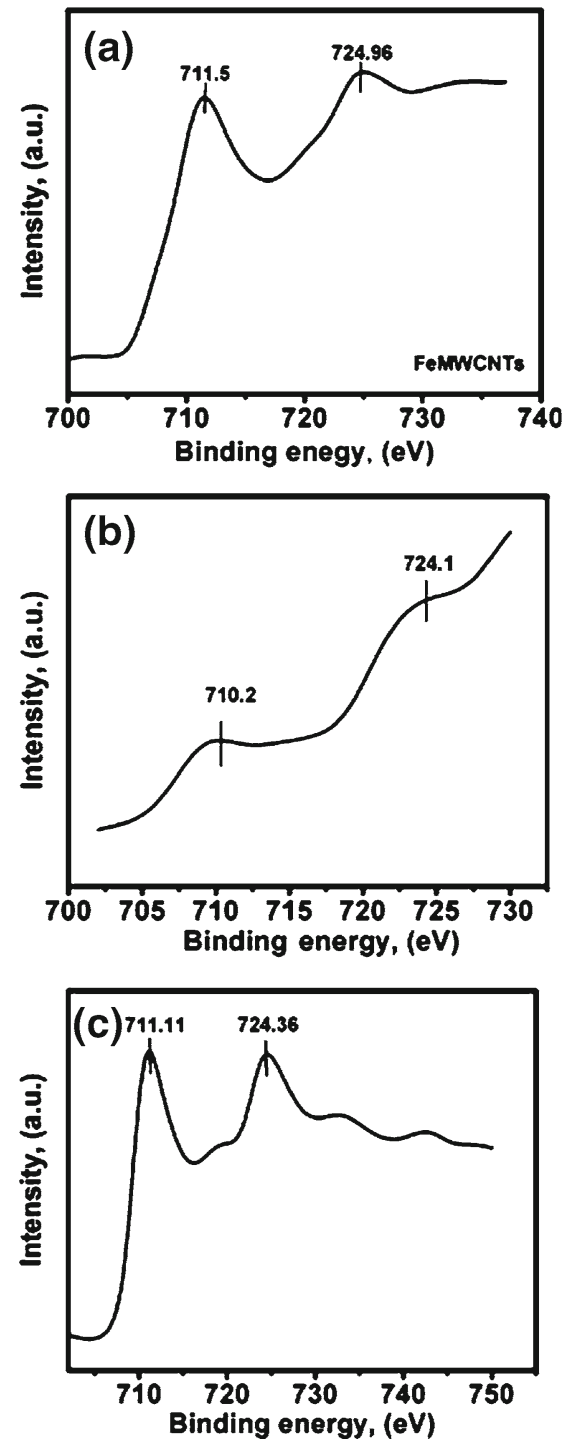

Figure 4. XPS for (a) Fe-MWCNTs, (b) Io-MWCNTs prepared by CCVD method and (c) Io-NPs prepared by the flame pyrolysis. magnetometer (VSM) measurements. The hysteresis plots of magnetization vs applied magnetic field and the values of coercivity, magnetization and retentivity are presented in the supporting information. The reported saturation magnetization (Ms) value for bulk $\gamma-\mathrm{Fe}_{2} \mathrm{O}_{3}$ is $73.5 \mathrm{emu} / \mathrm{g}$, which was found to be $42.9 \mathrm{emu} / \mathrm{g}$ in Io-NPs. Decreased in Ms value with respect to the size was attributed to the surface contribution (Zhou et al 2008). Still lesser Ms values for Fe-MWCNTs and Io-MWCNTs as compared with Io-NPs were observed, which could be explained on the basis of less proportion of $\mathrm{Fe}$ in the overall sample.
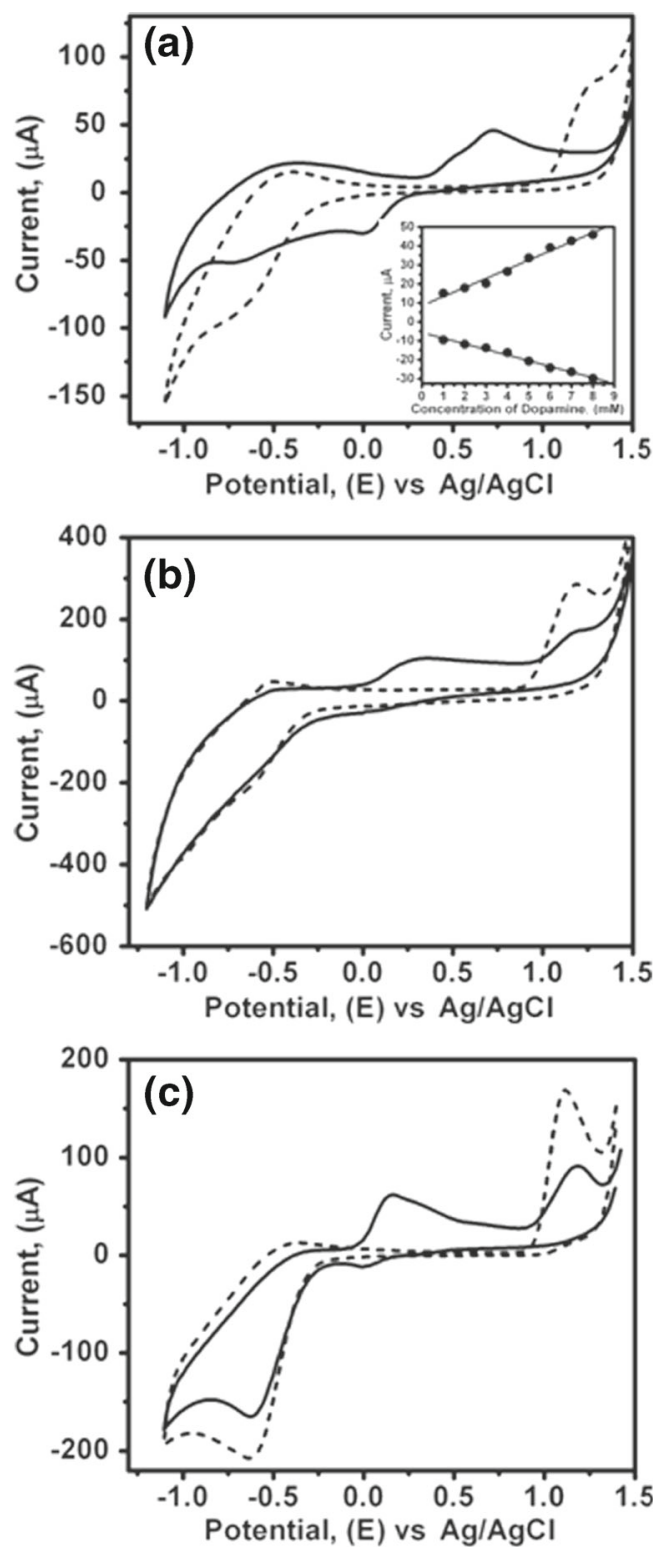

Figure 5. Cyclic voltammograms recorded at (a) $\mathrm{Fe}$ MWCNTs/MME, (b) Io-MWCNTs/MME and (c) Io-NPs in $0.2 \mathrm{mM} \mathrm{DA}$ in the presence of $0.1 \mathrm{M} \mathrm{KCl}$ solution in $\mathrm{Ar}$ atmosphere (scan rate is $50 \mathrm{mV} \mathrm{s}^{-1}$ ). Inset in (a) shows the linear relationship between redox current and concentration of DA. The control CV recorded for respective experiments are shown by the dotted line. 


\subsection{Voltammetric behaviour of dopamine and hydrogen peroxide on MWCNTS-MME}

A typical CV recorded at Fe-MWCNTs-MME in $0.2 \mathrm{mM}$ dopamine in the presence of $0.1 \mathrm{M} \mathrm{KCl}$ solution is shown in figure 5(a). A suppression of background redox activity along with the appearance of new anodic and cathodic peaks at 0.72 and $0.02 \mathrm{~V}$ respectively, were observed and found to be close to the one reported for DA (Xu et al 2004; Shultz et al 2007). The anodic peak at $0.72 \mathrm{~V}$ is assigned to the oxidation of DA and formation of dopaaminoquinone. The complimentary cathodic peak in the reverse scan at $0.02 \mathrm{~V}$ is assigned to the reduction of dopaaminoquinone. Figure 5(a) inset shows the plots of observed peak current values at $0.72 \mathrm{~V}$ over DA concentration, which fitted in the linear regression with $R^{2}=0.99$. This property supports the proposed use of Fe-MWCNTs-MME in the amperometric determination of DA.

$\mathrm{CV}$ recorded at Io-MWCNTs-MME in $0.2 \mathrm{mM} \mathrm{DA}$ is shown in figure 5(b). It gave the anodic peak for oxidation of DA at $0.29 \mathrm{~V}$. The similar measurements carried out on Io-NPs/MME (figure 5c), gave corresponding peak at $0.15 \mathrm{~V}$. Thus, based on these potential values, an ease of oxidation of DA on these samples are in the order of $\mathrm{Fe}$ MWCNTs $<$ Io-MWCNTs $<$ Io-NPs. The oxidation of DA is known to involve the molecular rearrangement followed by electron transfer and can be classified as inner sphere
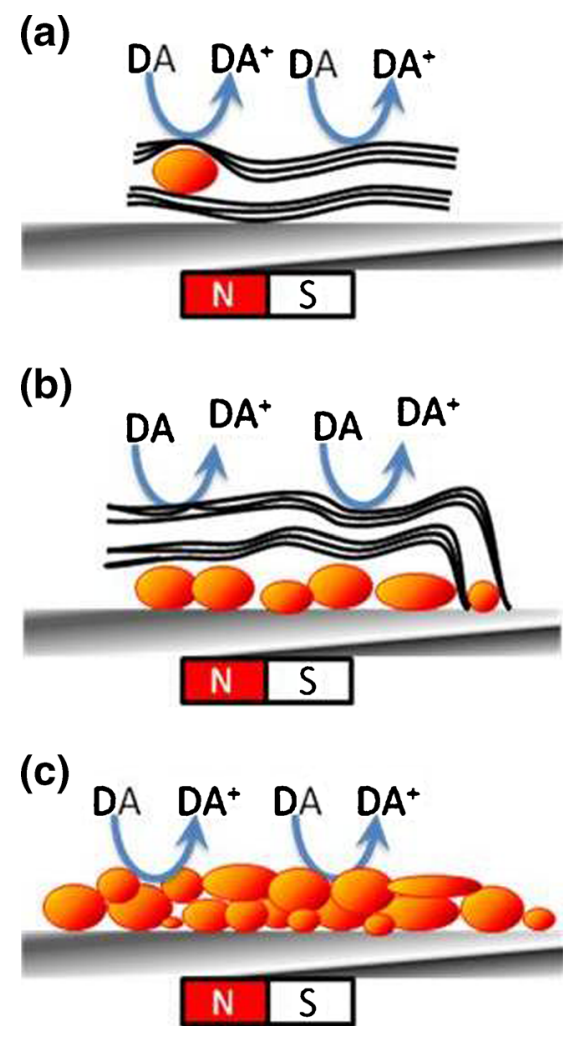

Figure 6. A schematic diagrams of (a) Fe-MWCNTs/MME, (b) Io-MWCNTs/MME and (c) Io-NPs/MME showing the position of iron oxide. electron transfer type. The electron transfer in such mechanism shows strong dependence on the nature of the electrode surface. In all three cases, the iron oxide is present in three distinct environments. In case of Fe-MWCNTs, the iron oxide particles are occluded inside the tube which suggests a strong interaction of iron oxide with MWCNTs. The reaction is likely taking place on MWCNTs "skin", which is under the strong influence of iron oxide particles present beneath. So, the iron oxide interacts indirectly with the surface adsorbed species "through" MWCNTs "skin". In case of Io-MWCNTs, it is rather physical mixture in which MWCNT has partly covered the iron oxide film. The
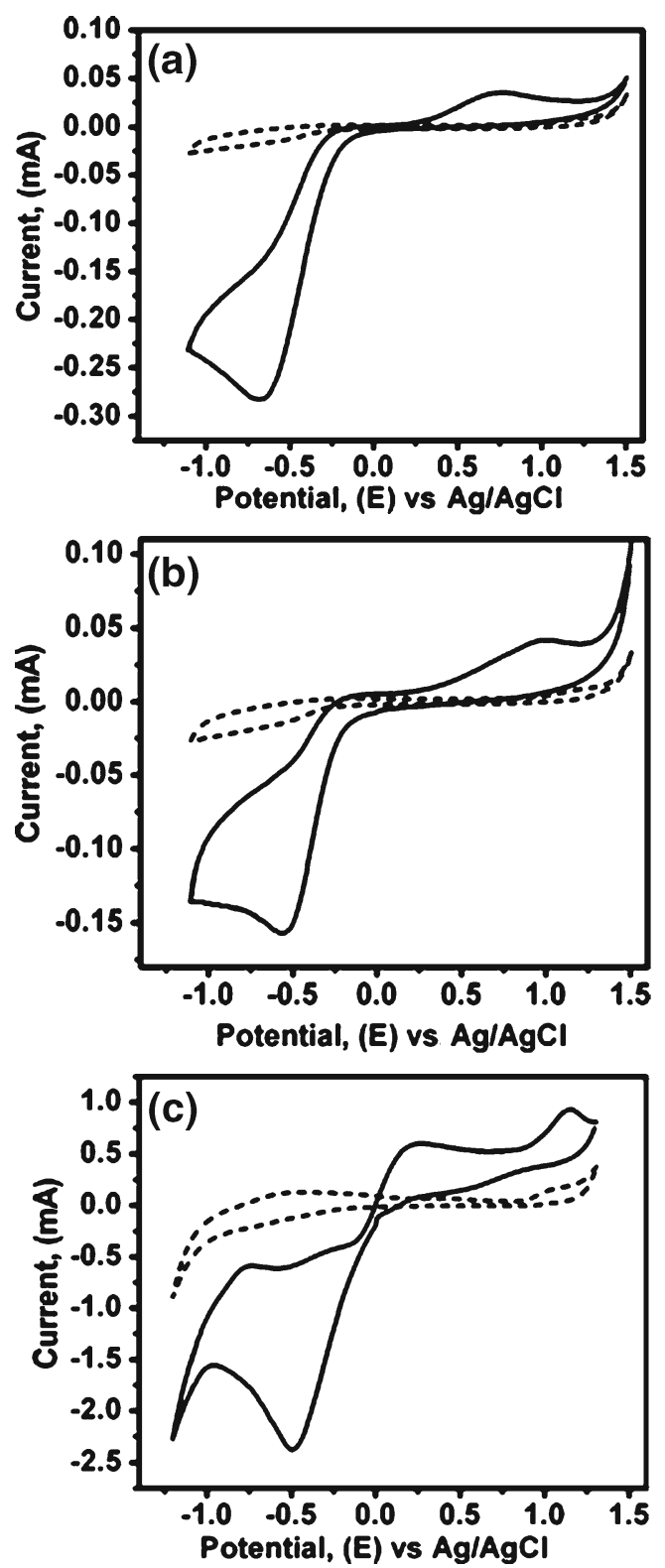

Figure 7. Cyclic voltammograms recorded at (a) $\mathrm{Fe}$ MWCNTs/MME, (b) Io-MWCNTs/MME and (c) Io-NPs/MME in $8 \mathrm{mM} \mathrm{HP}$ in presence of $0 \cdot 1 \mathrm{M} \mathrm{KCl}$ solution in $\mathrm{Ar}$ atmosphere. The control CV recorded for respective experiments are shown by the dotted line (scan rate is $50 \mathrm{mV} \mathrm{s}^{-1}$ ). 
reaction takes place on MWCNT surface as well as unexposed part of iron oxide particulate film. Thus influence of MWCNTs on the electrocatalytic activity of iron oxide is rather less compared to Fe-MWCNT case. Since, DA gets an opportunity of directly interacting with iron oxide, the reaction intermediates would get more stabilized which pushes the oxidation potential at less positive value. The iron oxide occluded in MWCNTs seems to be provided the surface in which the reaction intermediates has free energy towards higher side than MWCNTs or iron oxide alone. All the three cases are schematically described in figure 6.

\subsection{Electrochemical behaviour of hydrogen peroxide (HP)}

The performance of these three different electrodes was further tested for the hydrogen peroxide reduction. CVs recorded at Fe-MWCNTs-MME, Io-MWCNTs-MME and Io-NPs-MME in $8 \mathrm{mM} \mathrm{HP}$ in presence of $0.1 \mathrm{M} \mathrm{KCl}$ solution are shown in figures 7(a), (b) and (c), respectively. The profile for $\mathrm{CV}$ matched very well with the reported one (Sljukic et al 2006). On Fe-MWCNTs, it occurred at -0.67 V (refer figure 7a), which shifted to less negative potential values to -0.56 and $-0.49 \mathrm{~V}$ in case of Io-MWCNTs and Io-NPs, respectively (refer figures $7 \mathrm{~b}$ and $\mathrm{c}$ ). Thus, the thermodynamic feasibility of reduction of HP on these samples is in the order of Io-NPs $>$ Io-MWCNTs $>$ Fe-MWCNTs, which is in accordance with the one observed in case of DA oxidation. Again, in this case also the reduction of HP on MWCNTs is rather attributed to the iron oxide nanoparticles than MWCNTs itself. These results are parallel to the work reported by Compton and group (Sljukic et al 2006).

\section{Conclusions}

This study emphasis the role of impregnanted metal oxide in electrocatalytic behaviour of MWCNTs. The oxidation of dopamine and reduction of hydrogen peroxide is observed to be favourable on the iron oxide nanoparticles associated with MWCNTs. The novel magnetic electrodes facilitated the measurements without possible interference from binders, which more clearly distinguished activities of these catalytic centres. Thus, this study will be further helpful in the development of MWCNTs based modified electrodes for the detection of biomolecules viz. dopamine and hydrogen peroxide.

\section{Acknowledgements}

Financial support from DBT and ISRO-UoP, India, is gratefully acknowledged. KMS thanks the BRNS, CNQS-UoP for the Fellowship. VSJ thanks to CSIR for senior research fellowship. Authors thank the Physics Department, University of Pune, India, for SEM, XRD and VSM facilities.

\section{References}

Banks C E and Compton R G 2005 Analyst 1301232

Britto P J, Santhanam K S V, Rubio A, Alonso J A and Ajayan P M 1999 Adv. Mater. 11154

Chicharro M, Sanchez A, Bermejo E, Zapardiel A, Rubianes M D and Rivas G A 2005 Anal. Chim. Acta 54384

Fujii T, de Groot F M F, Sawatzky G A, Voogt F C, Hibma T and Okada K 1999 Phys. Rev. B59 3195

Gooding J J et al 2003 J. Am. Chem. Soc. 1259006

Inamdar S N and Haram S K 2006 J. Nanosci. Nanotechnol. 61

Jones C P, Jurkschat K, Crossley A, Compton R G, Riehl B L and Banks C E 2007 Langmuir 239501

Li W, Liang C, Zhou W, Qiu J, Zhou Z, Sun G and Xin Q 2003 J. Phys. Chem. B107 6292

Maogen Z and Waldemar G 2005 Anal. Chem. 773960

Musameh M, Wang J, Merkoci A and Lin Y 2002 Electrochem. Commun. 4743

Rubianes M D and Rivas G A 2003 Electrochem. Commun. 5689

Samant K M, Kapoor S and Haram S K 2007 Pramana J. Phys. 11054

Shultz M D, Reveles J U, Khanna S N and Carpenter E E 2007 J. Am. Chem. Soc. 1292482

Sljukic B, Banks C E and Compton R G 2006 Nano Lett. 61556

Valentini F, Amine A, Orlanducci S, Terranova M L and Palleschi G 2003 Anal. Chem. 755413

Wang J and Musameh M 2003 Anal. Chem. 752075

Wang J, Musameh M and Lin Y 2003 J. Am. Chem. Soc. 125 2408

Xu C, Xu K, Gu H, Zheng R, Liu H, Zhang X, Guo Z and Xu B 2004 J. Am. Chem. Soc. 1269938

Zhao Q, Gan Z and Zhuang Q 2002 Electroanalysis 141609

Zhou S-M, Zhang X-T, Gong H-C, Zhang B, Wu Z-S, Du Z-L and Wu S-X 2008 J. Phys: Condens. Matter 20075217 